\title{
A group of interacting yeast DNA replication genes
}

\author{
Kevin M. Hennessy, ${ }^{1}$ Angela Lee, ${ }^{2}$ Ellson Chen, ${ }^{2}$ and David Botstein ${ }^{1}$ \\ ${ }^{1}$ Department of Genetics, Stanford University, Stanford, California 94305 USA; ${ }^{2}$ Genentech, Inc., South San Francisco, \\ California 94080 USA
}

\begin{abstract}
Mutations in the cell-division-cycle genes $C D C 46$ and $C D C 47$ were originally isolated as suppressors of mutations in two other cell-division-cycle genes (CDC45 and CDC54). We found several combinations of mutations in these genes that result in allele-specific suppression and synthetic lethality, confirming that this set of genes forms a group of genetically interacting components. Here, we show that the other genes, like $C D C 46$, are all involved in an early step of DNA replication, possibly initiation of DNA synthesis. Mutants defective in each of the four genes exhibit high rates of mitotic chromosome loss and recombination. The mutants appear also to accumulate chromosome damage that can be detected by a novel chromosome electrophoresis assay. Conditional mutants in this group, under fully nonpermissive conditions, show cell-cycle arrest at the beginning of DNA synthesis; under less stringent conditions, some arrest later, in S-phase. The DNA sequence of the CDC46 gene indicates that the protein is a member of a new family of genes apparently required for DNA initiation, with family members now identified in Saccharomyces cerevisiae, Schizosaccharomyces pombe, and mouse cells.
\end{abstract}

[Key Words: Cell-division-cycle $(c d c)$ genes; DNA replication; DNA initiation; pulse-field CHEF gel; S. cerevisiae]

Received February 13, 1991; accepted March 12, 1991.

DNA replication is fundamental to the maintenance and growth of every eukaryotic organism. Saccharomyces cerevisiae is a particularly tractable model system for identifying the components of the DNA replication machinery because the distinctive growth pattern of this budding yeast allows cell-division-cycle $(c d c)$ phenotypes to be easily distinguished from defects in other cellular processes (Pringle and Hartwell 1981). Defects in DNA replication generally cause the cell cycle to arrest with general metabolism unaffected, so the mother cell eventually produces a large bud of equal size to itself. This phenotype has been used to identify conditional $c d c$ mutations from large random mutant collections established in several laboratories (Hartwell et al. 1970; Pringle et al. 1981; Moir et al. 1982).

One such collection of cold-sensitive mutations has been screened for cell-division-cycle defects at $17^{\circ} \mathrm{C}$ (Moir et al. 1982). Most of these mutations were in genes that had not been identified via screens of heat-sensitive mutant collections. Cold-sensitive mutations at two loci, $c d c 45-1$ and $c d c 54-1$, were found to arrest with a large bud and a single nucleus. Subsequent isolation of mutations in other loci that suppress the cell-cycle arrest at $17^{\circ} \mathrm{C}$ (second site revertants) revealed that a single cell-division-cycle gene (CDC46) could give rise to suppressors of mutations in two others (CDC45 and $C D C 54)$. Six independent suppressor mutations in $C D C 46$ were found that simultaneously make the cell heat sensitive at $37^{\circ} \mathrm{C}$ and arrest with a large bud and a single nucleus like $c d c 45$ and $c d c 54$ (Moir et al. 1982). A second gene, $C D C 47$, like CDC46, has been identified on the basis of a mutation $(c d c 47-1)$ that suppresses $c d c 45$ 1; this mutation also causes arrest with a large bud at $37^{\circ} \mathrm{C}$. Both the $c d c 46$ and $c d c 47$ suppressor mutations are remarkable in that each is itself a cell-division-cycle mutant with the same morphological arrest phenotype as that of the mutants that are suppressed by them. The phenotypes and origins of selected mutations in these four genes are summarized in Table 1.

We describe below the further characterization of this group of mutations and show that each interacts, at least genetically, with the others as would be expected for mutations in components of a protein complex. Evidence is also presented that all the members of this group arrest with a phenotype like $c d c 46-1$, which appears to be necessary for DNA initiation. In addition, the CDC46 product has a distinctive localization pattern that changes in coordination with the cell cycle; it is nuclear during interphase and cytoplasmically localized at other times in the cell cycle, suggesting that CDC46 and, by analogy, the other members of this group, are involved in regulation of DNA initiation (Hennessy et al. 1990).

\section{Results}

Genetic mapping of CDC46

To determine whether the CDC46 gene is among the 
Table 1. The CDC46 group

\begin{tabular}{lll}
\hline Allele & Phenotype & Method of isolation \\
\hline$c d c 45-1$ & cold-sensitive & general $\mathrm{Cs}^{-}$screen \\
$c d c 54-1$ & cold-sensitive & general $\mathrm{Cs}^{-}$screen \\
$c d c 46-1$ & heat-sensitive & suppressor of $c d c 45-1$ \\
$c d c 46-5$ & heat-sensitive & suppressor of $c d c 54-1$ \\
$c d c 47-1$ & heat-sensitive & suppressor of $c d c 45-1$ \\
\hline
\end{tabular}

$C D C$ genes that have already been identified and situated on the genetic map, we located its position on the genome by using a combination of physical and genetic methods. First, the chromosomal location was determined by hybridization of ${ }^{32} \mathrm{P}$-labeled DNA containing the gene to yeast chromosomes separated in a pulse-field CHEF gel as described in Materials and methods. The probe hybridized only with chromosome XII, so we crossed $c d c 46-1$ strains with strains that are marked over the length of chromosome XII (DBY4932 and DBY4933) (Johnson et al. 1987). Tetrad dissections of these crosses showed that $c d c 46$ is $29 \mathrm{cM}$ from $c d c 25$ and $\sim 50 \mathrm{cM}$ from ilv5 (Table 2). The distance between $c d c 25$ and ilv5 was confirmed to be $\sim 30 \mathrm{cM}$ (Johnson et al. 1987). Threefactor analysis verified that the relative gene order is $c d c 46-c d c 25-i I v 5$.

\section{Genetic mapping and assignment of the CDC54 gene}

Two different cold-sensitive mutations with a similar phenotype (arrest with a large bud) were found to be suppressed by different mutations in $c d c 46$ (Moir et al. 1982). One of these, CC30, had not been assigned a $c d c$ designation because the possibility remained that it is not a new locus but an additional allele of a previously identified $c d c$ locus. Hybridization of the cloned CC30 gene to CHEF-separated chromosomes indicated that it is situated on chromosome XVI. Genetic crosses with several centromere-linked markers (trp1, ura3, and leu2) indicated that $\mathrm{CC} 30$ is $\sim 6 \mathrm{cM}$ from its centromere (Table 3). A cross with the centromere XVI-linked marker aro7 (Mortimer et al. 1989) shows that the CC30 mutation is between aro7 and the centromere (Table 3). A second cross with $\operatorname{trp1}$, aro7, and CC3O (not shown) confirmed this order. The only gene known to map near this position is MAK6 (Mortimer et al. 1989), which is allelic to LTS5 (Ridley et al. 1984). We found that the coldsensitive lethal mutation CC30 complements mak6-1 (also a cold-sensitive allele) at $8^{\circ} \mathrm{C}$; thus, we believe that the two mutations define different genes.

Table 2. Mapping of the CDC46 gene

\begin{tabular}{lrccc}
\hline & \multicolumn{3}{c}{ Segregation } & \\
\cline { 2 - 4 } Gene pair & PD & NPD & TT & Map distance (cM) \\
\hline cdc46-cdc25 & 46 & 0 & 35 & 29 \\
ilv5-cdc25 & 25 & 0 & 43 & 31 \\
cdc46-ilv5 & 9 & 6 & 52 & $>50$ \\
\hline
\end{tabular}

Crosses between CC30 and those unmapped heat-sensitive loci that have a similar cell-cycle arrest phenotype (Pringle and Hartwell 1981) indicate that CC30 segregates independently of $c d c 20, c d c 30, c d c 23$, and $c d c 13$ (not shown; at least 10 tetrads were scored in each case). Thus, the CC 30 mutation defines a new cell-divisioncycle gene, CDC54, and CC30 will hereafter be referred to as $c d c 54-1$.

\section{Three genes that interact genetically with CDC46}

Because CDC46 was initially isolated as an extragenic suppressor of two cold-sensitive mutations, $c d c 45$ and $c d c 54$, we investigated its genetic interactions with the other members of this group. The results of this analysis are summarized in Figure 1, which shows instances of both synthetic lethality as well as suppression. We take up the evidence for each of these interactions in turn.

$\mathrm{CDC} 46$ and $\mathrm{CDC} 45$ show allele-specific suppression We determined this occurrence by readily recovering cdc46-1 cdc45-1 double mutants (13 double mutants of 12 tetrads dissected) that have the suppressed (i.e., cold resistant) phenotype, as shown originally by Moir et al. (1982). In contrast, cdc46-5 cdc45-1 double mutants, although readily recovered (15 doubles of 16 tetrads dissected), never showed suppression (i.e., the cold-sensitive phenotype characteristic of $c d c 45-1$ remains|.

CDC46 and CDC54 show allele-specific synthetic lethality as well as suppression In crosses of $c d c 46-5$ and cdc54-1 we confirmed suppression (Moir et al. 1982); double mutants were readily recovered (11 double mutants of 12 tetrads dissected) that all had the suppressed phenotype. In contrast, the cross of $c d c 46-1$ and $c d c 54-1$ resulted in recovery of no double mutants in 16 tetrads. To confirm this, the cross was carried out in the presence of a plasmid carrying the $C D C 46$ gene as well as the $U R A 3$ gene. In this case, the double mutant with the helper plasmid could be readily isolated (8 of 11 tetrads). However, when tested for the ability to lose the plasmid [i.e., ability to segregate progeny able to grow in the presence of 5-fluoro-orotic acid (Boeke et al. 1987)], the double mutant spores could not lose the plasmid, but progeny with either the $c d c 46-1$ or $c d c 54-1$ genes could.

CDC47 interacts differently with CDC46, CDC45, and CDC54 A cross between $c d c 47-1$ and $c d c 54-1$ shows synthetic lethality: No double mutants were recovered in 12 tetrads dissected. Likewise, a cross between $c d c 46$ 1 and $c d c 47-1$ also showed synthetic lethality (no double mutants recovered in 22 tetrads dissected). Here, again, the plasmid control was done, in which case the double mutants containing the helper plasmid could readily be recovered (not shown). In contrast, a cross between $c d c 47-1$ and $c d c 45-1$ shows suppression $(9$ of 11 tetrads, all with the suppressed phenotype), as expected from the results of Moir et al. (1982).

Taken together, these results indicate that the four genes in the group interact: Each of them gives rise to mutations that either suppress, are suppressed, or show 
Table 3. Mapping of the CDC54 gene

\begin{tabular}{|c|c|c|c|c|c|c|}
\hline \multirow[b]{2}{*}{ Gene pair } & \multicolumn{5}{|c|}{ Segregation } & \multirow[b]{2}{*}{ Map distance (cM) } \\
\hline & FD & SD & PD & NPD & $\mathrm{TT}$ & \\
\hline cdc54-CEN16 & 225 & 27 & & & & 6 \\
\hline aro7-CEN16 & 34 & 50 & & & & 30 \\
\hline$c d c 54-a r o 7$ & & & 42 & 1 & 45 & 29 \\
\hline
\end{tabular}

synthetic lethality in combination with one or more of the others. Allele specificity is observed, for both suppression and synthetic lethality. These types of combinatorial genetic effects are entirely consistent with the notion that these genes work together in the same pathway (Stearns and Botstein 1988).

\section{Mutations in the CDC46-related genes block DNA replication}

Mutations in the cell cycle can be ordered relative to the DNA replication step by determining the DNA content in individual cells as they arrest (Hartwell 1976; Pringle and Hartwell 1981). We blocked the progress of the cell cycle in a diploid strain (KHY210) homozygous for the cold-sensitive $c d c 45-1$ allele by shifting cells growing at their permissive temperature $\left(30^{\circ} \mathrm{C}\right)$ to two different nonpermissive temperatures $\left(15^{\circ} \mathrm{C}\right.$ and $\left.12^{\circ} \mathrm{C}\right)$. At both tem-

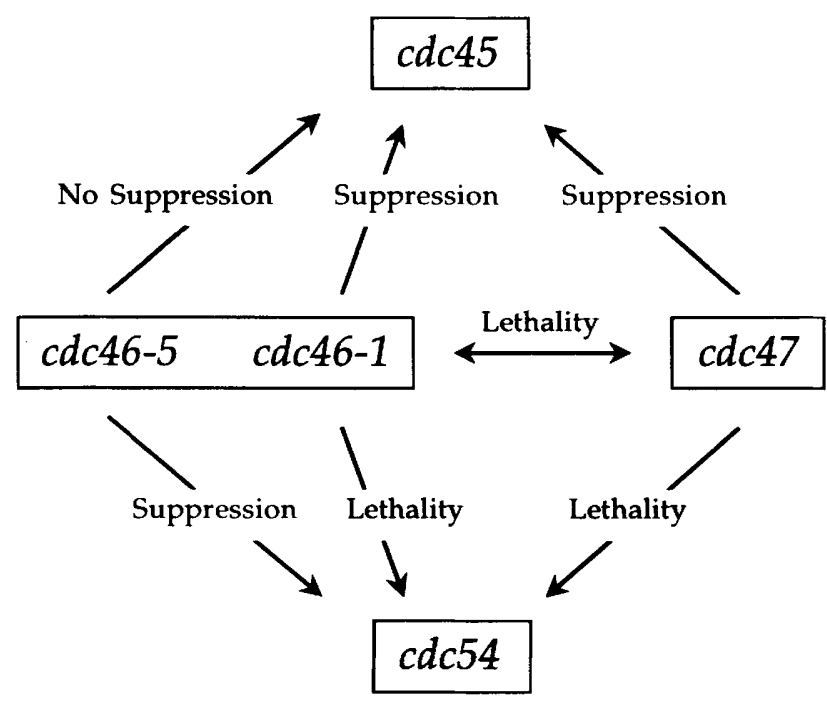

Figure 1. Four interacting genes required for completion of the cell cycle. Two were identified as cold-sensitive cell-cycle mutants (cdc45 and $c d c 54)$. $c d c 46$ and $c d c 47$ were isolated as suppressors of the cold-sensitive mutants. Mutant alleles of each were crossed to the other mutants within this group, and the phenotypes were determined. Some combinations create lethal phenotypes that neither parent exhibits individually. Alternatively, the $c d c 46-5$ and $c d c 54$ combination is both heat-sensitive and cold-sensitive. The other $c d c 46$ combinations suppress the cold-sensitive mutations as originally isolated. peratures, the cells arrested with a large bud during the first cycle after the shift into the cold. DNA content was determined by flow cytometry as described in Materials and methods after $\sim 1.5$ generation times at the nonpermissive temperature. Surprisingly, at $15^{\circ} \mathrm{C}$ the cells apparently complete DNA replication because every cell apparently contains two genome equivalents of DNA (Fig. 2B), whereas at $12^{\circ} \mathrm{C}$ the same strain arrested with only about one genome equivalent of DNA (Fig. 2C). Thus, even though $c d c 45-1$ causes an arrest with the same gross morphology at both $15^{\circ} \mathrm{C}$ and $12^{\circ} \mathrm{C}, 12^{\circ} \mathrm{C}$ appears to be a significantly more restrictive temperature as inferred by a tighter inhibition of DNA synthesis. Previous work, done with the same allele at $15^{\circ} \mathrm{C}$, suggested that this gene was required during mitosis, after DNA replication was finished (Moir et al. 1982). It is now apparent that $c d c 45-1$ is actually required early in DNA replication. We are left with the inference that even a partial block in DNA replication (as we found at $15^{\circ} \mathrm{C}$ ) results in the arrest of the cell cycle. Others have found regulatory mutations that may account for this arrest (see Discussion; Hartwell and Weinert 1989).

Figure 2 also shows that two other conditional mutants in the group, $c d c 46-1$ and $c d c 47-1$, caused cells to arrest with a single genomic equivalent of DNA. Diploid strains with homozygous mutations at either of these loci (DBY4921 and DBY4150), when shifted to nonpermissive temperature (in this case, $37^{\circ} \mathrm{C}$ ) from the permissive temperature $\left(26^{\circ} \mathrm{C}\right)$, accumulated large-budded cells and, like $c d c 45$, arrested with one genomic equivalent of DNA (Fig. 2E,F). The $c d c 46-1$ strain used carries a $c d c 46$ disruption on one chromosome (Hennessy et al. 1990) and the $c d c 46-1$ allele on the other, resulting in a very tight arrest at $37^{\circ} \mathrm{C}$. Similar results have been seen with a strain that has two copies of $c d c 46-1$ (Hennessy et al. 1990). An apparently less tight allele of $c d c 46, c d c 46-5$, manages to duplicate most of its genome before arresting, like $c d c 45-1$ at $15^{\circ} \mathrm{C}$ (not shown).

These results, especially the detailed similarities in leaky phenotype, buttress the notion that the four genes under study all cooperate in the same process, as indicated by the genetic interactions. The process is also identified by these results, namely, an early step in DNA replication. It is worth noting the results of Yan et al. (this issue) and Gibson et al. (1990) with the $\mathrm{mcm} 2$ and mcm3 mutations, which apparently affect origins of DNA replication and exhibit a phenotype very similar to that found for the leakier cdc46-5 and cdc45-1 phenotypes. 


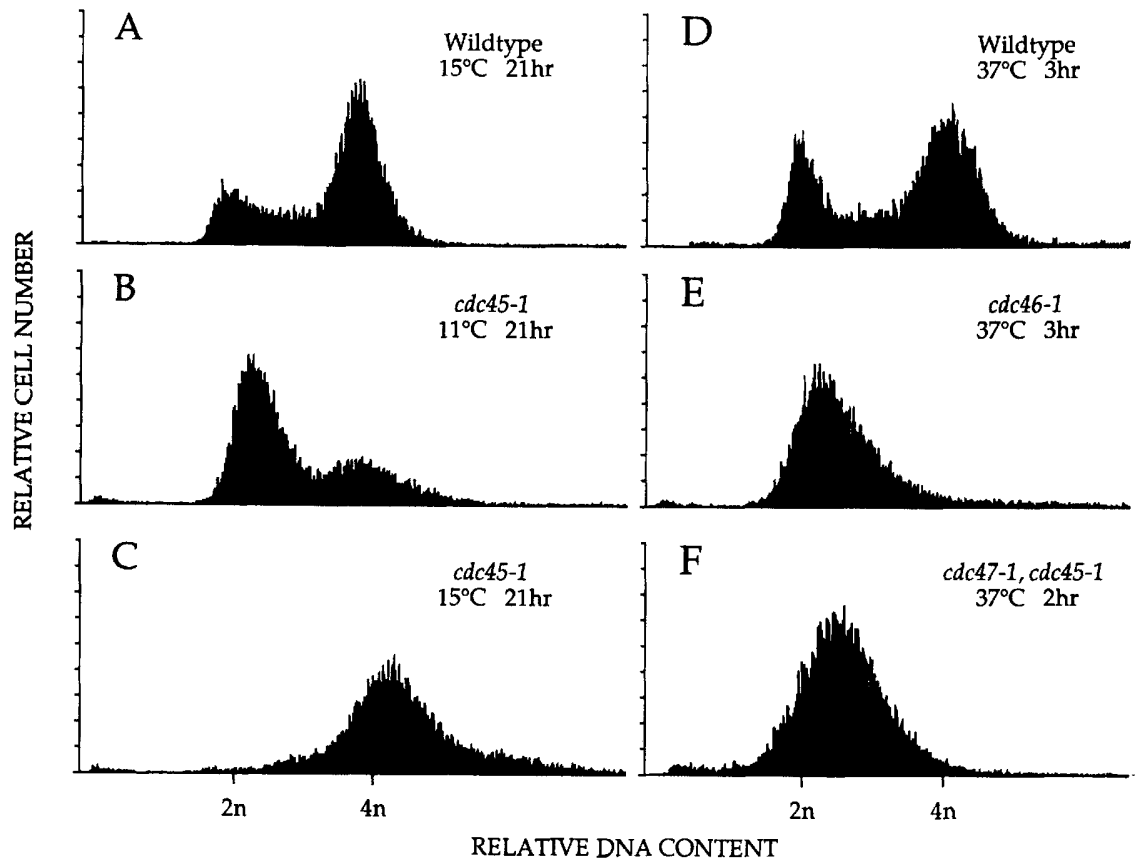

Figure 2. $C D C 45$ and $C D C 47$ are also required for DNA replication. The DNA content of individual $c d c 45$ cells was determined by flow cytometry. Cultures of both wild-type $(A)$ and $c d c 45$ diploid cells were shifted from a permissive temperature of $30^{\circ} \mathrm{C}$ to the nonpermissive temperatures of either $11^{\circ} \mathrm{C}(B)$ or $15^{\circ} \mathrm{C}(\mathrm{C})$. Both conditions resulted in $>95 \%$ arrest of the $c d c$ culture with large buds. In a separate experiment, the DNA content of wild-type $(D), c d c 46-1(E)$, and $c d c 47 c d c 45(F)$ homozygous diploid strains was determined after the indicated times at $37^{\circ} \mathrm{C}$. Although $c d c 45$ has no effect at this temperature, the $c d c 47$ arrest is improved in its presence. Both $c d c$ cultures contained $>90 \%$ large bud-arrested cells.
An additional piece of evidence for the interaction of these genes is shown in Figure 2F. The double mutant cdc47-1 cdc45-1 also shows an early block in DNA replication at $37^{\circ} \mathrm{C}$, the nonpermissive temperature for $c d c 47$. 1. As described above, cdc47-1 suppresses the cold-sensitive defect of $c d c 45-1$. This result is of interest because the $c d c 47-1$ allele alone has almost no inhibition of DNA replication under these conditions; therefore, the DNA replication block is another example of a conditional synthetic phenotype (Moir et al. 1982).

\section{Cell-cycle arrest causes chromosome damage}

One well-established method of distinguishing failure in mitosis from that in DNA replication is to assay the rate or frequency of chromosome recombination in cells that have been held up temporarily in the cell cycle by various mutations. Mutations of genes that are involved in DNA metabolism, such as those encoding DNA $\alpha$ polymerase (CDC17) (Carson 1987; Budd et al. 1989), DNA $\delta$-polymerase (CDC2) (Sitney et al. 1989), and DNA ligase (CDC9) (Johnston and Nasmyth 1978) all exhibit significantly elevated frequencies of mitotic recombination when put to this test (Hartwell and Smith 1985). Mutations in the mitotic apparatus, such as tub2, show an increase in the frequency of chromosome loss like the DNA synthesis mutations but show no increase in recombination (Huffaker et al. 1988).

We assayed the mitotic loss and recombination frequencies using standard heterozygous markers on chromosome V (Hartwell and Smith 1985; see also Materials and methods). Diploids homozygous for each of the various $c d c$ mutations were grown at permissive temperature and shifted to nonpermissive temperature for one and one-half generation times. Frequencies of chromo- some loss and recombination were normalized to a heterozygous $C D C 46 / c d c 46-1$ strain, which behaves identically to a homozygous $C D C 46 / C D C 46$ strain (Fig. 3). Significantly elevated recombination (10- to 100-fold) was evident in the three members of the CDC46-interacting group that we assayed. As expected, there is also an increase in the frequency of chromosome loss, which in this case is likely to be a secondary consequence of severe chromosome damage. In contrast, a mutation in the single $S$. cerevisiae $\beta$-tubulin gene causes an increase in chromosome loss but not recombination (Huffaker et al. 1988). It is important to note that these assays only observe the survivors of the initial arrest and that $c d c 46$ 1 causes a rapid loss of viability at the nonpermissive temperature. Thus, there is a drastic increase in the chromosome damage among the survivors of a $c d c 46, c d c 45$, and $c d c 54$ cell-cycle arrest.

\section{CHEF gel chromosome-mobility assay}

As a complement to the chromosome loss assay, we have developed a novel assay of chromosome damage that relies on a peculiarity of the CHEF and OFAGE pulse-field gel systems. In this experiment we arrested aliquots of an exponentially growing $c d c 46-1$ culture with various chemical treatments in addition to a $c d c 46$-induced arrest. The chromosomal DNA was run on a CHEF gel, blotted, and probed with a fragment of chromosome IV (Fig. 4, right). This probe was removed, and the same filter was rehybridized with a probe for chromosome III (Fig. 4, middle). The fraction of the chromosomes within the sample that can migrate into the gel differs with the arresting condition.

$\alpha$-Factor stops cells at the start of the cell cycle in $G_{1}$, well before DNA replication is initiated (Pringle and 
Figure 3. Mutations in the $C D C 46$ interacting group of genes result in high frequencies of chromosome loss and recombination. Diploid strains homozygous for the indicated gene were assayed for the frequency recombination and loss of chromosome V. Each strain was transiently held at its nonpermissive temperature for the time needed to complete 1.5 cell cycles and then returned to the permissive temperature for assaying. The values of chromosome loss and recombination for tub2-401 are from Huffaker et al. (1988).
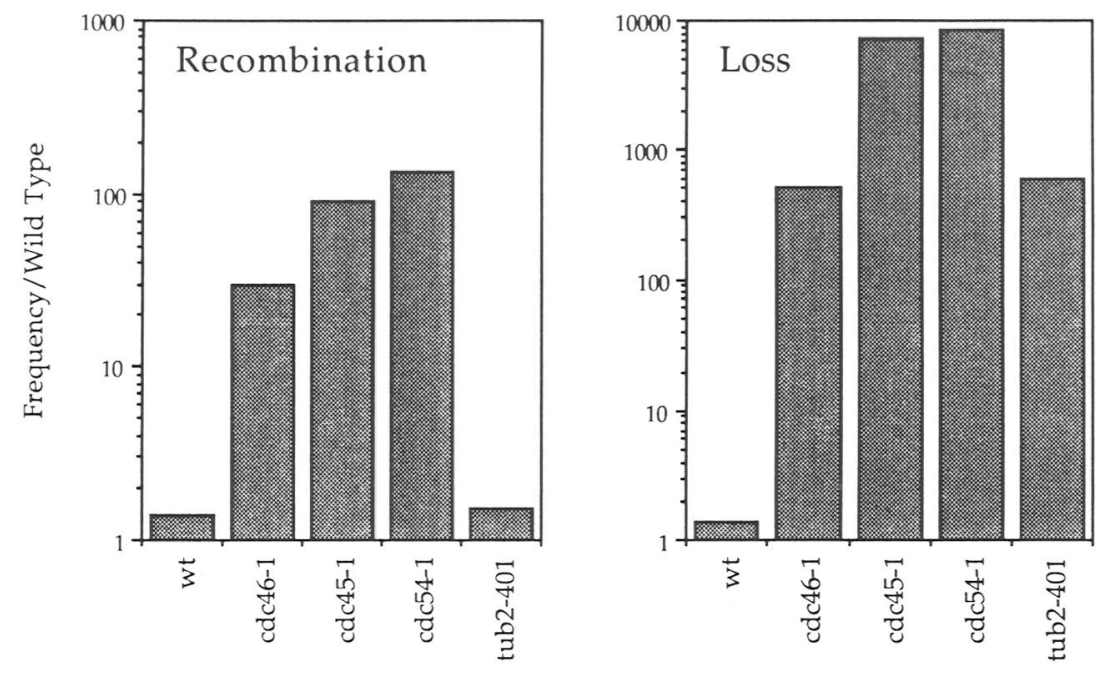

Hartwell 1981); and as shown in Figure 4, essentially all of the chromosomes within the sample migrate into the gel (lane 1). The same is true for cells that are arrested in mitosis with the drug nocodazole (lane 3). Mitotically arrested cells complete their DNA replication and contain a complete undamaged copy of the genome waiting to be divided between the daughter and mother (Huffaker et al. 1988). Thus, complete chromosomes, even when isolated from cell-cycle-arrested cells, are competent for CHEF gel migration.

Chromosomes are unable to enter the gel, however, when isolated from cells that have been arrested during

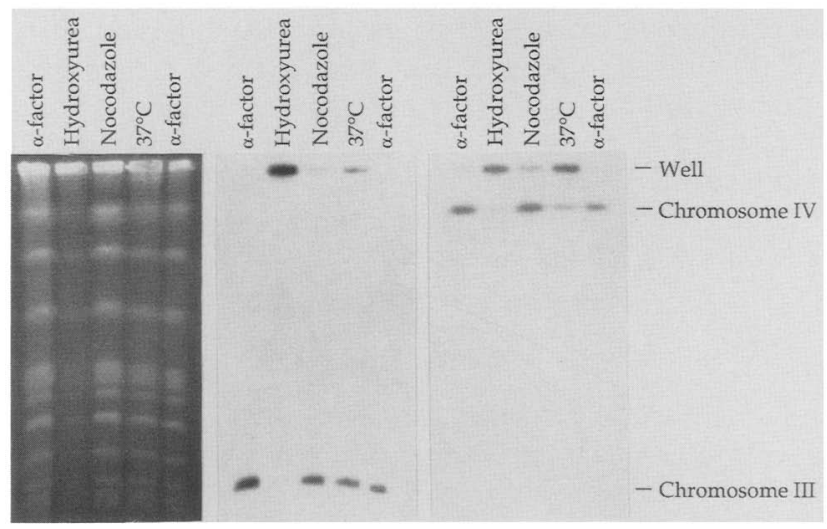

Figure 4. Chromosome abnormalities in CDC46 mutants. Aliquots of an actively growing $c d c 46-1$ strain have been arrested at various points in the cell cycle by using chemical inhibitors. Another aliquot of $c d c 46-1$ was held at the nonpermissive temperature at $37^{\circ} \mathrm{C}$ for $3 \mathrm{hr}$. The chromosomes of each aliquot were separated by CHEF and stained with ethidium bromide (left). The DNA was then acid-nicked, blotted, and hybridized with a ${ }^{32}$ P-labeled fragment of the SAC6 gene, which resides on chromosome IV) (right), or a labeled fragment of the LEU2 gene on chromosome III (middle). The top row of bands indicate where the sample was loaded and contain residual material that was not able to migrate into the gel.
DNA replication with hydroxyurea. This drug is thought to block nucleotide precursor synthesis (Slater 1973). Because pools of nucleotides within the cell initiate DNA replication, the cell can potentially arrest with "loops" of partially replicated DNA on the chromosome (Mariani and Shimke 1984). It is not surprising, then, that chromosomes isolated from a hydroxyurea-arrested strain are unable to enter the gel and remain within the well (Fig. 4, lane 2). This inhibition of migration affects all of the chromosomes equally with no regard to chromosome size.

Chromosomes from the $c d c 46$ strain arrested at $37^{\circ} \mathrm{C}$ for $3 \mathrm{hr}$ exhibit many similarities to those from a hydroxyurea-arrested cell (Fig. 4, lane 4). Inhibition of chromosome migration for the larger chromosomes, like chromosome IV, can be seen Figure 4 (right). However, unlike the hydroxyurea arrest, not all of the chromosomes are equally affected: The smaller chromosomes, like chromosome III, that migrate near the bottom of the gel (Fig. 4, middle) migrate nearly as well as those from the $\alpha$-factor or nocodazole controls. On the basis of ethidium bromide staining, all of the smaller chromosomes have been inhibited from running into the gel, rather than inhibition being specific to chromosome III.

Because $c d c 46-1$ causes cells to arrest with only one genomic equivalent of DNA, most chromosomes seen on the gel have not yet replicated (Hennessy et al. 1990; Fig. 2). Thus, $c d c 46-1$ causes damage to chromosomes early in the process of DNA replication in a way that is dependent on chromosome size. DNA initiation at several of the hundreds of DNA replication origins (Newlon 1988) would be more likely to occur on the larger chromosomes and provide a potential explanation for this phenomenon.

\section{Nucleotide sequence of the cdc46 gene}

By the strategy shown in Figure 5 , we determined the complete sequence of the CDC46 gene (Fig. 6). Two common promotor elements are evident: (1) a "TATA" 
consensus at base $392, \sim 60$ bases upstream of the open reading frame; and (2) a "CT" consensus at base 439 (Dobson et al. 1982). Three polyadenylation (AATAAA) sites are present, one 320 bases upstream of the open reading frame and two tandem sites at bases 2799 and 2953 , immediately after the open reading frame has terminated. The $\sim 2350$-base span between the promotor elements and polyadenylation sites is in accord with the 2300-base message detected on blotted agarose gels (Hennessy et al. 1990). One notably absent sequence motif is the branch site sequence (TACTAAC) required for splicing into this open reading frame from an upstream region (Langford et al. 1984). Consequently, this region has the recognized transcriptional signals consistent with its ability to encode the CDC46 gene.

An open reading frame spans from base 452 to 2792 with a consensus translational initiation site (A. AATG) at 468 (Dobson et al. 1982). The deduced protein sequence indicates that the CDC46 product is an $86-\mathrm{kD}$ acidic protein with a pI of 5.6. This is in rough agreement with the protein's apparent size of $95 \mathrm{kD}$ on the basis of SDS-PAGE. The nearly $10-\mathrm{kD}$ difference in apparent size between the deduced mass and the relative SDS-PAGE-determined mass is within the error attributable to anomalous migration found for other acidic proteins on SDS-PAGE (Takano et al. 1988).

Another intriguing feature of the protein sequence is a "PEST" site near the amino terminus (residues 5-29). PEST regions / the name coming from an abundance of prolines, aspartic, and glutamic acid, serine, and threonine residues) are found predominately in proteins with a high turnover rate (Rechsteiner 1988). Their removal can lead to an increased half-life in vivo (Cross 1988; Hadwiger et al. 1989). The PEST score for the CDC46 site is 3.4 , well within the range for proteins that are rapidly turned over. Most rapidly degraded proteins, but few stable proteins, have positive scores ranging from 0.3 to 25.4 (Rogers et al. 1986). An additional characteristic that $C D C 46$ shares with rapidly degraded proteins is an abundance of adjacent arginine residue pairs. $C D C 46$ has three arginine-arginine pairs, at residues 712,720 , and 734. These features predict that CDC46 might have a relatively short half-life within the cell.

Although most yeast proteins are not regulated in a cell-cycle-specific manner, a small group of genes, most of which are required for DNA synthesis, are known to be transcribed only during a brief period at the $G_{1} / S$ boundary. These genes have the small 6-bp sequence,
"ACGCGT" upstream of their promotors. Most of the genes in this group are thought to be involved with DNA synthesis or its regulation; these include $P O L I$ (DNA $\alpha$-polymerase), $c d c 2$ (DNA $\delta$-polymerase), $c d c 9$ (DNA ligase), SSB1 (single-stranded binding protein), CDC7 (protein kinase), $C D C 8$ (thymidylate kinase), and PRI1 and PRI2 (subunits of the DNA $\alpha$-polymerase) (Johnston et al. 1987; Foiani et al. 1989|. Although it is unlikely that this sequence is solely responsible and sufficient for cellcycle transcriptional regulation, it may play a role in conjunction with other regulatory factors. CDC46 also has this "cycle" box 310 bases upstream of the ATG. As we showed previously (Hennessy et al. 1990), the CDC46 gene is transcribed primarily during a single interval in the cell cycle, but unlike the others, during an $\alpha$-factor-induced $G_{1}$ arrest and immediately after $S$ phase. The significance of the presence of the ACGCGT sequence is thus unclear.

\section{Similar proteins exist in both Schizosaccharomyces} pombe and mammalian cells

Many of the proteins of $S$. cerevisiae have been found to be homologous to those of similar or identical function in other eukaryotic cells. The degree of this homology between yeast and mammals is generally on the order of $60 \%$ (Botstein and Fink 1988). Another yeast, Schizosaccharomyces pombe, is also a popular model system (Nurse 1990). Although both $S$. cerevisiae and $S$. pombe are yeasts, the degree of sequence divergence at the protein level between them is about the same as that between either yeast and mammalian cells (Beach et al. 1982; Schatz et al. 1986; Lee and Nurse 1987). Consequently, homologous, functionally similar proteins from two yeasts can potentially provide a good indication of the evolutionary and functional constraints acting on the protein.

We have identified immunologically related proteins in both $S$. pombe and mouse fibroblast cells. Affinitypurified antibodies isolated from two rabbits (Hennessy et al. 1990) react with the same size bands on immunoblots of total S. pombe protein (Fig. 7). On the basis of the migration in SDS-PAGE, the protein is $80 \mathrm{kD}$. This is slightly smaller than the $92-\mathrm{kD}$ protein seen in $S$. cerevisiae. A set of proteins is detected with the two rabbit antibodies, all in the same size range as the two yeast homologs. Not all of these bands react with both preparations of antibodies, but several at 100 and $88 \mathrm{kD}$ do react. This result implies that certain epitopes present

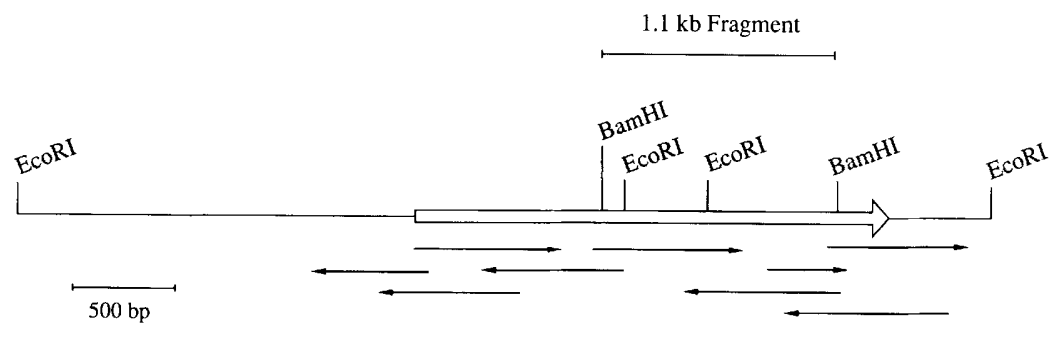

Figure 5. Restriction map of the CDC46 gene. The locations of restriction sites on the CDC46 gene are shown in relation to the open reading frame (open arrow). A summary of the sequencing strategy is presented below the map. 
TAGAAGTTTATACGGCCTTTATTTGTCAGTTATACTCCAGACTTAGTAAAAAATTCAATTAAATTCCAAACTGCTTACCCCAGATTTAGTGAATAAGAGCCCTTTATTC TACCCGCTTATCTCTGGATGTAAAACAAATAAACGCGTAATAGAGCAGTCAAACTAACAGCAGTAAACTGTCAATATCAAAAACGTAACAATTGATTCAAATCAGTTTATTCTAAGCCG CCTATGCCAGCCTTTAATTTTCTTCTCCTCCTCTTCACTCCCAGGTTAAGCCATTTAGTTTGTTGCTATGCGGTGTGTGGCGTGTTTCCCTTTTAGGAAAAGTGATTACTTGCAGTTTC GCGAAATCTCGAAGAATTTTTTCAACTTATTGAAAGAACATGAMTATACTTGTTAACGTGATTAAACGTTTTATCAGAACAACCTAAGAGTATCTCCTTGGTGAATTAGAAGCCAGATA MET SER PHE ASP ARG PRO GLU ILE TYR SER ALA PRO VAL LEU GLN GLY GLU SER PRO ASN ASP ASP ASP ASN THR GLU ILE ILE LYS SER
ATG TCA TTT GAT AGA CCG GAA ATA TAC AGT GCT CCT GTT TTA CAA GGA GAA TCT CCT AAC GAC GAT GAT AAT ACT GAA ATC ATA AAG TCC MET SER PHE ASP ARG PRO GLU ILE TYR SER ALA PRO VAL LEU GLN GLY GLU SER PRO ASN ASP ASP ASP ASN THR GLU ILE ILE LYS SER
ATG TCA TTT GAT AGA CCG GAA ATA TAC AGT GCT CCT GTT TTA CAA GGA GAA TCT CCT AAC GAC GAT GAT AAT ACT GAA ATC ATA AAG TCC ATG TCA TTT GAT AGA CCG GAA ATA TAC AGT GCT CCT GTT TTA CAA GGA GAA TCT CCT AAC GAC GAT GAT AAT ACT GAA ATC ATA AAG TCC PHE LYS ASN PHE ILE LEU GLU PHE ARG LEU ASP SER GLN PHE ILE TYR ARG ASP GLN LEU ARG ASN ASN ILE LEU VAL LYS ASN TYR SER PHE LYS ASN PHE ILE LEU GLU PHE ARG LEU ASP SER GLN PHE ILE TYR ARG ASP GLN LEU ARG ASN ASN ILE LEU VAL LYS ASN TYR SER
TTT AAG AAT TTC ATT TTG GAG TTC AGA CTT GAC TCG CAA TTT ATT TAC AGA GAT CAG TTA AGG AAC AAC ATC CTT GTG AAG AAT TAT TCT 70 70
LEU THR VAL ASN MET GLU HIS LEU ILE GLY TYR ASN GLU ASP ILE TYR LYS LYS LEU SER ASP GLU PRO SER ASP ILE ILE PRO LEU PHE
TTA ACG GTT AAC ATG GAG CAT TTG ATC GGA TAT AAC GAA GAC ATA TAT AAG AAA CTA TCA GAC GAA CCT TCA GAT ATC ATT CCA TTA TTC TTA ACG GTT AAC ATG GAG CAT TTG ATC GGA TAT AAC GAA GAC ATA TAT AAG AAA CTA TCA GAC GAA CCT TCA GAT ATC ATT CCA TTA TTC GLU THR ALA ILE THR GLN VAL ALA IYS ARG ILE SER ILE LEU SER ARG ALA GLN SER ALA ASN ASN ASN ASP LYS ASP PRO GLU ASN THR GAA ACC GCG ATC ACA CAA GTG GCT AAA AGG ATA AGT ATT CTA AGC AGA GCT CAA TCT GCT AAT AAC AAT GAC AAA GAT CCA GAA AAT ACT 130

SER MET ASP THR ASP SER LEU LEU LEU ASN SER LEU PRO THR PHE GLN LEU ILE LEU ASN SER ASN ALA ASN GLN ILE PRO LEU ARG ASP $\begin{array}{llll}160 & 170 & 180\end{array}$ LEU ASP SER GLU HIS VAL SER LYS ILE VAL ARG LEU SER GLY ILE ILE ILE SER THR SER VAL LEU SER SER ARG ALA THR TYR LEU SER TTG GAT TCT GAA CAC GTC TCC AAG ATT GTC CGT TTA TCA GGT ATT ATA ATA TCC ACG TCA GTT ITA TCT TCC CGT GCC ACG TAC CTT TCT 1902000 ILE MET CYS ARG ASN CYS ARG HIS THR THR SER ILE THR ILE ASN ASN PHE ASN SER ILE THR GLY ASN THR VAL SER LEU PRO ARG SER ATA ATG TGC AGA AAT TGC AGA CAC ACA ACA TCA ATA ACA ATC AAC AAT TTC AAT TCT ATC ACA GGC AAT ACC GTC AGT TTA CCA CGT TCT $\begin{array}{ll}220 & 230 \\ 2 & \end{array}$ CYS LEU SER THR ILE GLU SER GLU SER SER MET ALA ASN GLU SER ASN ILE GLY ASP GLU SER THR LYS LYS ASN CYS GLY PRO ASP PRO TGC TTA TCT ACG ATT GAG AGT GAA TCT TCT ATG GCA AAC GAG TCG AAT ATT GGT GAT GAA TCG ACC AAG AAA AAT TGT GGA CCT GAT CCA TYR ILE ILE ILE HIS GLU SER SER LYS PHE ILE ASP GLN GLN PHE LEU LYS LEU GLN GLU ILE PRO GLU LEU VAL PRO VAL GLY GLU MET TAT ATT ATT ATC CAT GAG TCT TCA AAG TTT ATT GAT CAA CAG TTT TTA AAA TTA CAG GAA ATC CCA GAA CTG GTT CCA GTA GGT GAG ATG 280 TYR IEU THR ASN LYS VAL ILE PRO GLY THR ARG VAL THR ILE VAL GLY ILE TYR SER ILE PRO ARG ASN LEU THR MET THR CYS ASP ARG TYR LEU THR ASN LYS VAL ILE PRO GLY THR ARG VAL THR ILE VAL GLY ILE TYR SER ILE CCT AGA AAC TTA ACA ATG ACT TGT GAC CGA TAC CTA ACA AAC AAA GIT ATS CCI GGT NCG AGA GTC ACT ATA GTA GGI AIT TAT ICC ATC TYR ASN SER LYS ASN GLY ALA GLY SER GLY ARG SER GLY GLY GLY ASN GLY GLY SER GLY VAL ALA ILE ARG THR PRO TYR ILE LYS ILE TAT AAT TCT AAA AAT GGT GCC GGA TCC GGA AGG AGC GGG GGT GGA AAT GGA GGA AGT GGT GTT GCT ATT AGA ACA CCT TAT ATC AAA ATA $340 \quad 350 \quad 360$ LEU GLY ILE GLN SER ASP VAL GLU THR SER SER ILE TRP ASN SER VAL THR MET PHE THR GLU GLU GLU GLU GLU GLU PHE LEU GLN LEU TTA GGT ATT CAG TCC GAT GTA GAA ACC TCC TCT ATT TGG AAT TCA GTA ACT ATG TTT ACT GAG GAG GAA GAA GAG GAA TTT CTA CAG CTA 3300
390 SER ARG ASN PRO LYS LEU TYR GLU ILE LEU THR ASN SER ILE ALA PRO SER ILE PHE GLY ASN GLU ASP ILE LYS LYS ALA ILE VAL CYS AGT AGA AAC CCG AAG CTT TAT GAA ATT TTG ACC AAC TCT ATT GCC CCC TCT ATT TTT GGT AAT GAA GAT ATA AAA AAA GCC ATT GTA TGT $400 \quad 410 \quad 420$ LEU LEU MET GLY GLY SER LYS LYS ILE LEU PRO ASP GLY MET ARG LEU ARG GLY ASP ILE ASN VAL LEU LEU LEU GLY ASP PRO GLY THR LEU LEU MET GLY GLY SER LYS LYS ILE LEU PRO ASP GLY MET ARG LEU ARG GLY ASP ILE ASN VAL LEU LEU LEO GLY ASP PRO GLY TAR
TTA TTG ATG GGT GGT TCC AAG AAG ATA TTA CCC GAT GGT ATG AGG TTA AGA GGT GAT ATC AAT GTA CTA TTA TTA GGT GAT CCA GGT ACC 430
440 ALA LYS SER GLN LEU LEU LYS PHE VAL GLU LYS VAL SER PRO ILE ALA VAL TYR THR SER GLY LYS GLY SER SER ALA ALA GLY LEU THR $\begin{array}{rl}460 & 470 \quad 480\end{array}$ 460
470
ARG GLU PHE TYR LEU GLU GLY GLY ALA MET VAL LEU ALA ASP GLY GLY VAL VAL CYS ILE ASP ALA SER VAL GLN ARG ASP PRO MET THR ARG GLU PHE TYR LEU GLU GLY GLY ALA MET VAL LEU ALA ASP GLY GLY VAL VAL CYS ILE ASP
GCC AGT GTA CAA AGA GAT CCG ATG ACA AGA GA TTT TAT TTG GAA GGT GGT GCT ATG GTG CTT GCC GAT GGT GGT GTT GTA TGC ATC GAT GCC AGT GTA CAA AGA GAT CCG ATG ACA AGA GAA TTT TAT TTG GAA GGT GGT GCT ATG GTG CTT GCC GAT GGT GGT GTT GTA TGC ATC GAT
500 GLU PHE ASP LYS MET ARG ASP GLU ASP ARG VAL ALA ILE HIS GLU ALA MET GLU GLN GLN THR ILE SER ILE ALA LYS ALA GLY ILE THR GAA TTC GAT AAA ATG AGA GAT GAA GAT AGA GTG GCC ATT CAT GAA GCT ATG GAG CAG CAA ACA ATC TCC ATC GCA AAA GCT GGT ATC ACT $520 \quad 530 \quad 540$ THR VAL LEU ASN SER ARG THR SER VAL LEU ALA ALA ALA ASN PRO ILE TYR GLY ARG TYR ASP ASP LEU LYS SER PRO GLY ASP ASN ILE ACA GTG CTA AAT TCT AGA ACT AGT GTT TTA GCG GCT GCT AAT CCG ATA TAC GGC CGG TAT GAT GAT TTG AAG TCT CCT GGT GAC AAC ATT $550 \quad 560 \quad 570$ ASP PHE GLN THR THR ILE LEU SER ARG PHE ASP MET ILE PHE ILE VAL LYS ASP ASP HIS ASN GLU GLU ARG ASP ILE SER ILE ALA ASN ASP PHE GLN THR THR ILE LEU SER ARG PHE ASP MET ILE PHE ILE VAL LYS ASP ASP HIS ASN GLU GLU ARG ASP ILE SER ILE ALA ASN
GAT TTC CAA ACT ACT ATT TTA TCC CGT TTT GAT ATG ATT TTT ATT GTT AAG GAT GAC CAT AAT GAA GAA CGT GAT ATT TCA ATA GCT AAC $580 \quad 590 \quad 600$ HIS VAL ILE ASN ILE HIS THR GLY ASN ALA ASN ALA MET GLN ASN GLN GLN GLU GLU ASN GLY SER GLU ILE SER ILE GLU LYS MET LYS CAC GTT ATT AAT ATT CAT ACA GGA AAT GCT AAT GCT ATG CAA AAC CAA CAA GAG GAA AAT GGC AGT GAA ATT AGT ATT GAA AAG ATG AAA

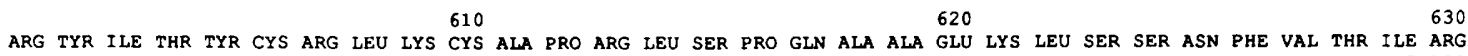
ARG TYR ILE THR TYR CYS ARG LEU LYS CYS ALA PRO ARG LEU SER PRO GLN ALA ALA GLU LYS LEU SER SER ASN PHE VAL THR ILE ARG
CGT TAC ATT ACG TAT TGT AGA TTG AAA TGT GCA CCA AGA CTT TCA CCG CAG GCC GCT GAA AAA CTG TCA TCG AAC TTC GTC ACC ATT AGG 640 650 6 660 LYS GLN LEU LEU ILE ASN GLU LEU GLU SER THR GLU ARG SER SER ILE PRO ILE THR ILE ARG GLN LEU GLU ALA ILE ILE ARG ILE THR AAG CAA TTA TTA ATC AAC GAA TTA GAG TCA ACG GAA AGG TCG TCT ATT CCA ATT ACC ATT CGT CAA TTA GAA GCT ATT ATT AGA ATA ACA 670
GLU SER LEU ALA LYS LEU GLU LEU SER PRO ILE ALA GLN GLU ARG HIS VAL ASP GLU ALA ILE ARG LEU PHE GLN ALA SER THR MET ASP GAA TCA TTA GCC AAG TTA GAA TTA AGT CCT ATT GCA CAG GAG AGA CAT GTT GAC GAA GCT ATT AGA TTG TTT CAA GCT TCC ACA ATG GAC $700 \quad 710 \quad 720$ ALA ALA SER GLN ASP PRO ILE GLY GLY LEU ASN GLN ALA SER GLY THR SER LEU SER GLU ILE ARG ARG PHE GLU GLN GLU LEU LYS ARG
GCA GCG TCT CAG GAT CCA ATT GGC GGC TTA AAT CAA GCA AGC GGA ACA TCT TTG TCA GAA ATC CGT CGT TTT GAA CAA GAA CTA AAA AGA GCA GCG TCT CAG GAT CCA ATT GGC GGC TTA AAT CAA GCA AGC GGA ACA TCT TTG TCA GAA ATC CGT CGT TTT GAA CAA GAA CTA AAA AGA ARG LEU PRO ILE GLY TRP SER THR SER TYR GLN THR LEU ARG ARG GLU PHE VAL ASP THR HIS ARG PHE SER GLN LEU ALA LEU ASP LYS AGA TTA CCT ATA GGC TGG TCT ACT TCT TAT CAA ACT TTG AGG AGA GAA TTT GTA GAT ACA CAT AGA TTT TCT CAA TTA GCA CTG GAT AAG 760 ALA LEU TYR ALA LEU GLU LYS HIS GLU THR ILE GLN LEU ARG HIS GLN GLY GLN ASN ILE TYR ARG SER GLY VAL OP
GCC TTA TAT GCC CTA GAG AAG CAT GAA ACA ATT CAA TTG AGA CAC CAG GGA CAG AAT ATT TAC AGA AGT GGT GTA TGA CAACAATAABGTCTT AGCTTGACGCCTTTTCTACTTGTTTGCATGTAATGAATCTAAAAATTCGCTACAAATACTTCATTATTATATTTCTATATAAATTCTGTAAACGAAATTATACTTTTTGAGGTTTCAT CTGCGTCTCATATTCTTTTTGTATAATAAACGCACAGACTTGATATGACCGTCAATCAAAATAATTCAAAGCTTCTTAAAAAAGAAATTGAAATCAAAGACATACGAAAGAAATTAGAA

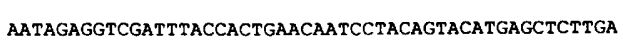

Figure 6. (See facing page for legend.) 
on the CDC46 protein (CDC46p) have been highly conserved over the large evolutionary time scale that divides these yeasts and mammal cells.

A search of a yeast protein data base (M. Goebl, pers. comm.) identified a gene in $S$. pombe that is similar to CDC46p along its entire length. This protein is the product of the NDA4 gene, which was first identified by a cell-division-cycle mutation (Toda et al. 1983; M. Yanagida, pers. comm.). An alignment of the two proteins is shown over a 45-residue stretch (Fig. 8) as an example of the remarkable degree of homology between these two proteins. The predicted mass of the NDA4 protein is within the error expected, on the basis of SDS-PAGE migration, for the band in the immunoblot above $(M$. Yanagida, pers. comm.). Because both cdc46 in Saccharomyces and NDA4 in Schizosaccharomyces cause cellcycle defects at their restrictive temperature, their functions might well also have been conserved.

Another sequence, from mouse cells, also shows a high level of homology $(71 \%)$ to CDC46p. This short, 45-residue protein sequence is deduced from a small stretch of cDNA sequence isolated from a cDNA library (Breier et al. 1988). The sequence now appears to have come from a cloning artifact and is best considered an anonymous mouse sequence in the DNA sequence data base; we have confirmed that this DNA is present in the mouse genome by polymerase-chain-reaction experiments (not shown) that produced a fragment of the predicted size. Thus, there are known genes in both mouse and $S$. pombe cells that could encode the proteins homologous to CDC46p seen on immunoblots.

\section{Discussion}

The group of genes that we have studied were first thought to be associated because of the observation of Moir et al. (1982) that different alleles of $c d c 46$ could suppress mutations in $c d c 45$ and $c d c 54$. We have extended the observation of these interactions by showing further allele specificity and hitherto unsuspected synthetic lethality interactions among the members of the group. These interactions are summarized in Figure 1. The genetic evidence linking this group of genes is of such a nature as to suggest strongly the possibility that all of these genes are functional parts of a single process or pathway.

Given the level of genetic interactions observed between $C D C 46, C D C 45, C D C 54$, and $C D C 47$, it is no coincidence that the four genes have such similar terminal phenotypes at their nonpermissive temperatures. The common arrest phenotypes include the following. (1) A common terminal arrest morphology. Each arrests with a single large bud, a mitotic spindle assembled, and nucleus at the bud neck. (2) Evidence of chromosome

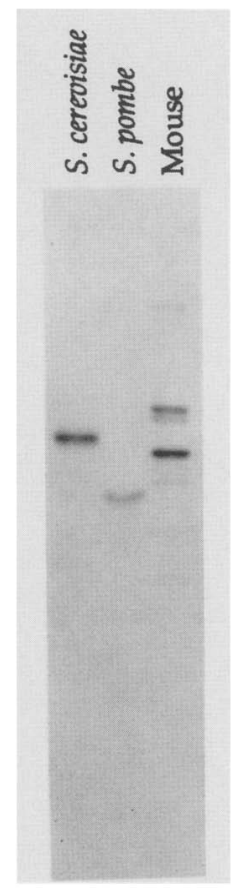

Figure 7. Antibodies against $S$. cerevisiae $C D C 46$ product recognize similar size proteins in other species. Shown is an immunoblot of total cellular protein from the divergent yeasts $S$. cerevisiae and $S$. pombe or the mouse L-cell line after being reacted with affinity-purified anti-CDC46p antibodies.

damage as a result of the cell-cycle arrest. (3) Incomplete DNA replication, with the degree of completion being dependent on the tightness of the conditional phenotype.

The prototypical mutation of the group is $c d c 46-1$. We showed previously (Hennessy et al. 1990) that the CDC46 function is first required before DNA elongation. To account for the cell-cycle-dependent pattern of nuclear staining of the CDC46 protein, we suggested that CDC46 might function in coordinating the initiation of new DNA synthesis (Hennessy et al. 1990). We assumed that the firing of hundreds of DNA replication origins must be organized in such a way that each fires once, and only once, during each round of DNA replication. The process could then be thought to be governed by a factor that enters the nucleus at the end of mitosis and is maintained within the nucleus until the next cell-division cycle begins (Blow and Laskey 1988).

DNA initiation is clearly a complex process that must be completed once started for viability to be maintained (Newlon 1988). Nevertheless, mutations in this process might be expected to produce cells with unusual "incomplete" DNA replication phenotypes. Our observations indicate that the different nonpermissive tem-

Figure 6. Nucleotide sequence of the $C D C 46$ gene. The DNA sequence of the CDC46 gene and the deduced protein sequence of the product are indicated. Two polyadenylation signals are underlined at the 3 ' end of the sequence, and the putative cell cycle regulatory motif is underlined at the $5^{\prime}$ end. 
Hennessy et al.

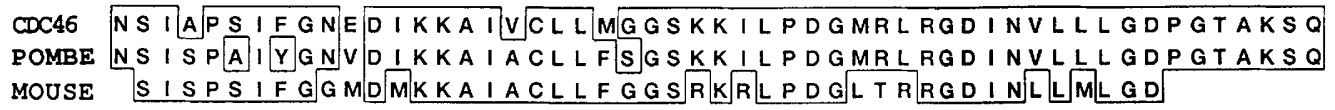

Figure 8. Sequence similarities between $S$. cerevisiae $C D C 46, S$. pombe, and mouse proteins. A short segment of amino acids is shown, derived from the DNA sequence of CDC46 (CDC46), the $S$. pombe gene NDA4 (pombe), and an anonymous cDNA mouse sequence (mouse). Identical residues between two or more sequences are boxed.

peratures with the $c d c 45-1$ mutant can be interpreted in this light. The least permissive condition leads to an early arrest with little or no net DNA synthesis. The defect must not interfere with elongation once a cell has started to replicate its DNA. In the next cell cycle, there then is no DNA initiation and cells finally arrest with a large bud. The block imposed on $c d c 45-1$ by the less stringent nonpermissive condition causes cells to arrest with DNA replication essentially being complete at the level of detection provided by the flow cytometry; the interpretation is that some origins of replication fire while others do not. The DNA replication is more or less completed with fewer than normal initiations, the chromosomes are damaged or otherwise altered because of this, and a secondary regulatory process (such as that envisioned for RAD9; Hartwell and Weinert 1989) is responsible for the terminal cell-cycle arrest with a large bud.

Yan et al. (this volume) present new results on a set of mutations in the genes MCM2 and MCM3, identified as having a connection to the yeast origins of replication (Maine et al. 1984; Gibson et al. 1990). The phenotypes of these mutations at their restrictive condition closely resemble the leakier phenotype of $c d c 45-1$, lending considerable independent support to the interpretation that the CDC46-related group is involved in initiation of DNA replication. This similarity extends to the hyperrecombination and chromosome loss phenotypes as well. An even stronger argument for the connection derives from the comparison of the predicted amino acid sequences of the $M C M 2$ and $M C M 3$ products with the product of $C D C 46$. The similarity is on the order of $60 \%$ identity within a 400 -amino-acid domain.

Other mutations in the replication apparatus, specifically those in the DNA $\alpha$-polymerase that is encoded by the $C D C 17$ gene, can give a similar intermediate arrest phenotype at a semipermissive temperature (Carson 1987). Thus, it appears that DNA replication mutations can generally be difficult to detect via flow cytometry assays, until a fully nonpermissive temperature is established with a tight allele. Subsequent differentiation of DNA initiation defects from DNA elongation defects requires further ordering of the functions relative to known elongation defects. This type of ordering has been used to establish that CDC46 is required for DNA initiation rather than DNA elongation (Hennessy et al. 1990).

The DNA damage evident in $c d c 46-1$ cells at both the level of elevated mitotic recombination and the inability of chromosomes to migrate on CHEF gels is a result of these defects in DNA replication. DNA damage, although typically associated with a DNA elongation defect, could just as easily be caused by initiation of DNA replication at only a fraction of the origins that would normally be used. The fact that damage is more severe in the larger chromosomes is consistent with this model, because the smaller chromosomes might not have any of their origins firing (and, thus, may not be damaged) and the larger chromosomes will be more likely to have at least one origin fired and thus be damaged. It is important in this context to remember that these CHEF assessments were done under fully nonpermissive conditions.

Recently, the number of DNA sequences has increased, and it has become possible to identify homologous genes in other organisms as well as discover new gene families. We have provided evidence for members of this gene family being present in mammalian as well as other yeast eukaryotic cells. Affinity-purified antibodies recognize a family of proteins, all similar in size to the yeast proteins, in cultured mouse cells. Furthermore, we have made the serendipitous identification of an anonymous mouse sequence that, when translated, is $70 \%$ identical to $C D C 46$ over its entire 40-residue length. This homology is within the region conserved between all the known members of this DNA initiation family, including MCM2 and MCM3. Thus, there is evidence for a newly identified family of proteins involved in the early steps of DNA replication.

\section{Materials and methods}

Strains and media

Yeast media and genetic manipulations have been described by Sherman et al. (1974). S. cerevisiae strains used in this study are listed in Table 4. The cold-sensitive $c d c$ mutant CC30 (Moir et al. 1982), which arrests with a large bud at $18^{\circ} \mathrm{C}$, has been named $c d c 54-1$. The $c d c 46-1$ allele is described as the rA18-95 isolate, and $c d c 46-5$ as the rCC30-161 (Moir et al. 1982). Bacterial media were as described in Davis et al. (1980). The Escherichia coli strain that was used for routine cloning, $\mathrm{HBl0l}$ (DB1142), has the genotype $l e u^{-}$pro $^{-} t h r^{-} h s d R^{-} h s d M^{-}$ rec $A^{-}$.

\section{Determination of yeast cell DNA content}

Whole diploid cells of the yeast strains were prepared for the fluorescence-activated cell sorter by staining with propidium iodide (Hutter and Eipel 1978). Briefly, cells were ethanol-fixed and treated overnight with $0.1 \%$ RNase followed by $5 \mathrm{mg} / \mathrm{ml}$ of proteinase $\mathrm{K}$ prior to incubation in $5 \mathrm{mg} / \mathrm{ml}$ of propidium iodide. The propidium iodide was removed immediately prior to running each sample through a cytofluorograph system (Ortho Diagnostic Systems, Inc., Westwood, MA). 
Table 4. Genotype and source of strains

\begin{tabular}{|c|c|c|}
\hline Strain & Genotype & Source \\
\hline DBY1034 & MAT $\alpha$ his4-539 lys2-801 ura3-52 & this lab \\
\hline DBY4150 & MATa/MAT $\alpha$ ade2-1/ADE2 his4-619/HIS4 cdc45-1/cdc45-1 cdc47-1/cdc47-1 & this study \\
\hline DBY4921 & $\begin{array}{l}\text { MATa/MAT } \mathbf{\alpha} \text { ura3-52/ura3-52 leu2-3,112/LEU2 ade2-1/ADE2 lys2-801/LYS2 his4-619/HIS4 } \\
\text { cdc46-1/cdc46:URA3:cdc46 }\end{array}$ & this study \\
\hline DBY4922 & $\begin{array}{l}\text { MATa/MAT } \mathbf{a r a 3 - 5 2 / u r a 3 - 5 2 ~ l e u 2 - 3 , 1 1 2 / L E U 2 ~ a d e 2 - 1 / A D E 2 ~ l y s 2 - 8 0 1 / L Y S 2 ~ h i s 4 - 6 1 9 / H I S 4 ~} \\
\text { cdc46:URA3:cdc46/CDC46 }\end{array}$ & this study \\
\hline DBY4933 & MATa cdc3-1 ura3 leu2 gal2 RDN1::LEU2 & this lab \\
\hline DBY4932 & MAT $\alpha$ ilv5-1 asp5 ura4 & this lab \\
\hline KH203 & $\begin{array}{l}\text { MATa/MAT } \alpha \text { ura3-52/URA3 hom3/HOM3 } \text { can }^{\mathrm{R}} / \text { CAN }^{\mathrm{S}} \text { lys2-801/LYS2 his4-619/HIS4 } \\
\text { cdc54-1/cdc54-1 }\end{array}$ & this study \\
\hline KH210 & $\begin{array}{l}\text { MATa/MAT } \alpha \text { ura3-52/URA3 hom3/HOM3 } \text { can }^{\mathrm{R}} / \text { CAN }^{\mathrm{S}} \text { lys2-801/LYS2 his4-619/HIS4 } \\
\text { cdc45-1/cdc45-1 }\end{array}$ & this study \\
\hline KH195 & MATa/MATa ura3-52/URA3 hom3/HOM3 $\mathrm{can}^{\mathrm{R}} / C A N^{\mathrm{S}}$ lys2-801/LYS2 his4-619/HIS4 & this study \\
\hline KH208 & 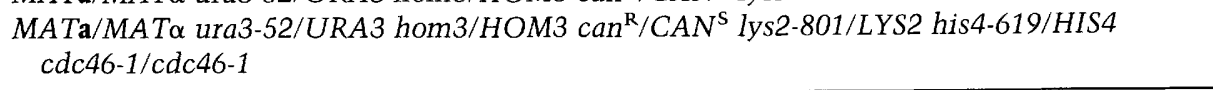 & this study \\
\hline
\end{tabular}

\section{DNA sequencing}

The EcoRI 2.4-kb, EcoRI 1.4-kb, and BamHI 1.1-kb fragments (see Fig. 5\} were isolated, ligated separately into sequencing vectors pUC119 and pUCl18, and cloned in the SR101 bacterial strain. Single-stranded phage were sequenced by using the method of Sanger (Sanger et al. 1977). Besides the universal primers, six insert-specific primers were used to provide overlapping sequence across the cloning restriction sites. Computer analysis of the sequence was done with the Genentech package of software (C. Watanabe, pers. comm.). PEST scores were determined by using the scoring methodology of Rechsteiner (1988) in the PEST-SCORE algorithm. Data bases searched were GenBank release 63 and EMBL release 22.

\section{Chromosome loss and recombination}

Diploid strains, homozygous for either $c d c 46-1$ (KH208), cdc451 (KH210), or cdc54-1 (KH203) were assayed for mitotic recombination and loss of chromosome $\mathrm{V}$ by using a slightly modified version of the assay of Hartwell and Smith (1985). Each strain is marked on one of the two chromosome Vs with a recessive canavanine-resistance allele on one side of the centromere and the serine, threonine auxotrophy (hom 3 ) on the other. Cells that have lost the wild-type $\mathrm{Can}^{\mathrm{s}}$ gene are selected after a brief incubation at the restrictive temperature $\left(3 \mathrm{hr}\right.$ at $37^{\circ} \mathrm{C}$ for $c d c 46$ and $12 \mathrm{hr}$ at $15^{\circ} \mathrm{C}$ for $c d c 45$ or $c d c 54$ ). These clones (200) were then screened for hom 3 auxotrophy. An isogenic strain without a cell-cycle defect, as well as a heterozygous cdc46-1/CDC46 strain, were assayed in the same way and the data have been normalized to the frequency of chromosome loss and recombination in the heterozygous $c d c 46-1$ strain.

\section{Chromosomal mapping}

Chromosomal DNA samples were prepared as described (Holm et al. 1989/ from S. cerevisiae strains DBY1034, DBY2370, and DBY2371 and separated on $1 \%$ agarose slab gels in $0.5 \times$ TBE buffer at $11^{\circ} \mathrm{C}$ for $21 \mathrm{hr}$ at $7.6 \mathrm{~V} / \mathrm{cm}$ with a switching time of 80 sec using a CHEF gel apparatus. The gel was transferred to GeneScreen Plus (NEN Research, Boston, MA) and hybridized to a ${ }^{32}$ P-labeled CDC46 1.1-kb BamHI fragment probe (Fig. 5) using standard methodology (Maniatis et al. 1982; Sambrook et al. 1989).

\section{Chromosome OFAGE migration assay}

Yeast chromosomal DNA samples were prepared from arrested cultures as described previously (Holm et al. 1989). Cells were lysed in an agar matrix with high concentrations of zymolyase T100, $\beta$-mercaptoethanol, and $0.5 \mathrm{M}$ EDTA and then treated with proteinase $\mathrm{K}$ and detergent. Finally, the agarose plugs were washed with $50 \mathrm{~mm}$ EDTA. The samples were then applied to a $1 \%$ agarose gel slab and electrophoresed for $21 \mathrm{hr}$ at $11^{\circ} \mathrm{C}$ with a field strength of $7.6 \mathrm{~V} / \mathrm{cm}$ and a switching time of $80 \mathrm{sec}$ in $0.5 \times$ TBE (Maniatis et al. 1982). The gel was subsequently stained for $30 \mathrm{~min}$ with ethidium bromide and photographed. The separated chromosomes were then acid-nicked and transferred to GeneScreen Plus. The blot was probed with ${ }^{32}$ P-labeled SAC6 and LEU2 DNA (Andreadis et al. 1984; Adams et al. 19891 .

\section{Acknowledgments}

We thank Tim Stearns for discussions about the CHEF gel assay, and Kai-uwe Froehlich for initial isolation of the CDC46 gene. We are indebted to Mitsuhiro Yanagida, Bik-kwoon Tye, and Steve Kearsey for sharing their unpublished results and to Mark Goebl for locating the sequence homologies between MCM2, MCM3, CDC46, NDA4, and CDC21.

The publication costs of this article were defrayed in part by payment of page charges. This article must therefore be hereby marked "advertisement" in accordance with 18 USC section 1734 solely to indicate this fact.

\section{References}

Adams, A.E.M., D. Botstein, and D.G. Drubin. 1989. A yeast actin-binding protein is encoded by SAC6, a gene found by suppression of an actin mutation. Science 243: 231-233.

Andreadis, A., Y.-P. Hsu, M. Hermodson, G.B. Kohlhaw, and P. Schimmel. 1984. Yeast LEU2: Repression of mRNA levels by leucine and primary structure of the gene product. $/$. Biol. Chem. 259: 8059-8062.

Beach, D., B. Durkacz, and P. Nurse. 1982. Functionally homologous cell cycle control genes in fission yeast and budding yeast. Nature 300: 706-709.

Blow, J. and R. Laskey. 1988. A role for the nuclear envelope in controlling DNA replication within the cell cycle. Nature 332: 546-548. 
Boeke, I.D., J. Trueheart, G. Natsoulis, and G.R. Fink. 1987. 5-Fluoro-orotic acid as a selective agent in yeast molecular genetics. Methods Enzymol. 154: 164-175.

Botstein, D. and G.R. Fink. 1988. Yeast: An experimental organism for modern biology. Science 240: 1439-1443.

Breier, G., G. Dressler, and P. Gruss. 1988. Primary structure and develpmental expression pattern of $H o x$ 3.1, a member of the murine Hox 3 homeobox gene cluster. EMBO I. 7: 1329-1336.

Budd, M.E., K.D. Wittrup, J.E. Bailey, and J.L. Campbell. 1989. DNA polymerase I is required for premeiotic DNA replication and sporulation but not for X-ray repair in Saccharomyces cerevisiae. Mol. Cell. Biol. 9: 365-376.

Carson, M.J. 1987. CDC17, the structural gene for DNA polymerase I of yeast: Mitotic hyperrecombination and effects on telomere metabolism, Ph.D. thesis. University of Washington, Seattle.

Cross, F.R. 1988. DAF1, a mutant gene affecting size control, pheromone arrest, and cell cycle kinetics of Saccharomyces cerevisiae. Mol. Cell. Biol. 8: 4675-4684.

Davis, R., D. Botstein, and J. Roth. 1980. Advanced bacterial genetics. Cold Spring Harbor Laboratory, Cold Spring Harbor, New York.

Dobson, M., M. Tuite, N. Roberts, A. Kingsman, S. Kingsman, R. Perkins, S. Conroy, B. Dunbar, and L. Fothergill. 1982. Conservation of high efficiency promoters in Saccharomyces cerevisiae. Nucleic Acids Res. 10: 2625-2637.

Foiani, M., C. Santocanale, P. Plevani, and G. Lucchini. 1989. A single essential gene, PRI2, encodes the large subunit of DNA primase in Saccharomyces cerevisiae. Mol. Cell. Biol. 9: 3081-3087.

Gibson, S.I., R.T. Surosky, and B. Tye. 1990. The phenotype of the minichromosome maintenance mutant $\mathrm{mcm} 3$ is characteristic of mutants defective in DNA replication. Mol. Cell. Biol. 10: 5707-5720.

Hadwiger, J.A., C. Wittenberg, H.E. Richardson, M. Barros Lopes, and S.I. Reed. 1989. A family of cyclin homologs that control the G1 phase in yeast. Proc. Natl. Acad. Sci. 86: 6255-6259.

Hartwell, L.H. 1976. Sequential function of gene products relative to DNA synthesis in the yeast cell cycle. I. Mol. Biol 104: 803-817.

Hartwell, L.H., J. Culotti, and B. Reid. 1970. Genetic control of the cell-division cycle in yeast. I. Detection of mutants. Proc. Natl. Acad. Sci. 66: 352--359.

Hartwell, L.H. and D. Smith. 1985. Altered fidelity of mitotic chromosome transmission in cell cycle mutants of S. cerevisiae. Genetics 110: 381-395.

Hartwell, L.H. and T.A. Weinert. 1989. Checkpoints: Controls that ensure the order of cell cycle events. Science 246: 629635.

Hennessy, K.M., C.D. Clark, and D. Botstein. 1990. Subcellular localization of yeast $C D C 46$ varies with the cell cycle. Genes \& Dev. 4: 2252-2263.

Holm, C., T. Stearns, and D. Botstein. 1989. DNA topoisomerase II must act at mitosis to prevent nondisjunction and chromosome breakage. Mol. Cell. Biol. 9: 159-168.

Huffaker, T.C., J.H. Thomas, and D. Botstein. 1988. Diverse effects of $\beta$-tubulin mutations on microtubule formation and function. I. Cell Biol. 106: 1997-2010.

Hutter, K.J. and H.E. Eipel. 1978. Flow cytometric determinations of cellular substances in algae, bacteria, molds and yeast. Antonie Leeuwenhoek I. Microbiol. Serol. 44: 269282.

Johnson, D.I., C.W. Jacobs, J.R. Pringle, L.C. Robinson, G.F. Carle, and M.V. Olson. 1987. Mapping of the Saccharomyces cerevisiae $\mathrm{CDC} 3, \mathrm{CDC} 25$, and $\mathrm{CDC} 42$ genes to chromosome XII by chromosome blotting and tetrad analysis. Yeast 3: 243-253.

Johnston, L.H. and K.A. Nasmyth. 1978. S. cerevisiae cell cycle mutant $c d c 9$ is defective in DNA ligase. Nature 274: 891894.

Johnston, L.H., J.H. White, A.L. Johnson, G. Lucchini, and P. Plevani. 1987. The yeast DNA polymerase I transcript is regulated in both the mitotic cell cycle and in meiosis and is also induced after DNA damage. Nucleic Acids Res. 15: 5017-5030.

Langford, C.J., F.-J. Klinz, C. Donath, and D. Gallwitz. 1984. Point mutations identify the conserved, intron-contained TACTAAC box as an essential splicing signal sequence in yeast. Cell 36: 645-653.

Lee, M.G. and P. Nurse. 1987. Complementation used to clone a human homolog of the fission yeast cell cycle control gene cdc2. Nature 327: 31-35.

Maine, G., P. Sinha, and B. Tye. 1984. Mutants of S. cerevisiae defective in the maintenance of minichromosomes. Genetics 106: 365-385.

Maniatis, T., E.F. Fritsch, and J. Sambrook. 1982. Molecular cloning: A laboratory manual. Cold Spring Harbor Laboratory, Cold Spring Harbor, New York.

Mariani, B.D. and R.T. Schimke. 1984. Gene amplification in a single cell cycle in chinese hamster ovary cells. I. Biol. Chem. 259:1901-1910.

Moir, D. and D. Botstein. 1982. Determination of the order of gene function in the yeast nuclear division pathway using cs and ts mutants. Genetics 100: 565-577.

Moir, D., S.E. Stewart, B.C. Osmond, and D. Botstein. 1982. Cold-sensitive cell-division-cycle mutants of yeast: Properties and pseudoreversion studies. Genetics 100: 547-563.

Mortimer, R.K., D. Schild, C.R. Contopoulou, and J.A. Kans. 1989. Genetic map of Saccharomyces cerevisiae, 10th ed. Yeast 5: 321-403.

Newlon, C.S. 1988. Yeast chromosome replication and segregation. Microbiol. Rev. 52: 568-601.

Nurse, P. 1990. Universal control mechanism regulating onset of M-phase. Nature 344: 503-508.

Pringle, J.R. and L.H. Hartwell. 1981. The Saccharomyces cerevisiae cell cycle. In The molecular biology of the yeast Saccharomyces: Life cycle and inheritance led. J.N. Strathern, E.W. Jones, and J.R. Broach), pp. 97-142. Cold Spring Harbor Laboratory, Cold Spring Harbor, New York.

Rechsteiner, M. 1988. Regulation of enzyme levels by proteolysis: The role of PEST regions. Adv. Enz. Reg. 627: 135-151.

Ridley, S.P., S.S. Sommer, and R.D. Wickner. 1984. Superkiller mutations in Saccharomyces cerevisiae suppress exclusion of M2 double-stranded RNA by L-A-HN and confer cold sensitivity in the presence of M and L-A-HN. Mol. Cell. Biol. 4: 761-770.

Rogers, S., R. Wells, and M. Rechsteiner. 1986. Amino acid sequences common to rapidly degraded proteins: The PEST hypothesis. Science 234: 364-368.

Sambrook, J., E.F. Fritsch, and T. Maniatis. 1989. Molecular cloning: A laboratory manual. Cold Spring Harbor Laboratory Press, Cold Spring Harbor, New York.

Sanger, F., S. Nicklen, and A.R. Coulson. 1977. DNA sequencing with chain-terminating inhibitors. Proc. Natl. Acad. Sci. 74: 5463-5467.

Schatz, P.J., L. Pillus, P. Grisafi, F. Solomon, and D. Botstein. 1986. Two functional $\alpha$-tubulin genes of the yeast Saccharomyces cerevisiae encode divergent proteins. Mol. Cell. Biol. 6: 3711-3721.

Sherman, F., G. Fink, and C. Lawrence. 1974. Methods in yeast 
genetics. Cold Spring Harbor Laboratory, Cold Spring Harbor, New York.

Sitney, K.C., M.E. Budd, and J.L. Campbell. 1989. DNA polymerase III, a second essential DNA polymerase, is encoded by the S. cerevisiae CDC2 gene. Cell 56: 599-605.

Slater, M.L. 1973. Effect of reversible inhibition of deoxyribonucleic acid synthesis on the yeast cell cycle. I. Bacteriol. 113: $263-270$.

Stearns, T. and D. Botstein. 1988. Unlinked noncomplementation: Isolation of new conditional-lethal mutations in each of the tubulin genes of Saccharomyces cerevisiae. Genetics 119: 249-260.

Takano, E., M. Maki, H. Mori, M. Hatanaka, T. Marti, K. Titani, R. Kannagi, T. Ooi, and T. Murachi. 1988. Pig heart calpastatin: Identification of repetitive domain structures and anomalous behavior in polyacrylamide gel electrophoresis. Biochemistry 27: 1964-1972.

Toda, T., K. Umesono, A. Hirata, and M. Yanagida. 1983. Coldsensitive nuclear division arrest mutants of the fission yeast Schizosaccharomyces pombe. I. Mol. Biol. 168: 251-270. 


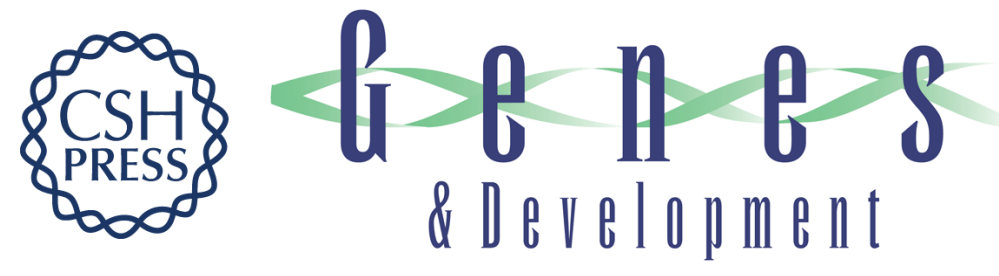

\section{A group of interacting yeast DNA replication genes.}

K M Hennessy, A Lee, E Chen, et al.

Genes Dev. 1991, 5:

Access the most recent version at doi:10.1101/gad.5.6.958

References This article cites 43 articles, 24 of which can be accessed free at: http://genesdev.cshlp.org/content/5/6/958.full.html\#ref-list-1

\section{License}

Email Alerting

Receive free email alerts when new articles cite this article - sign up in the box at the top Service right corner of the article or click here.

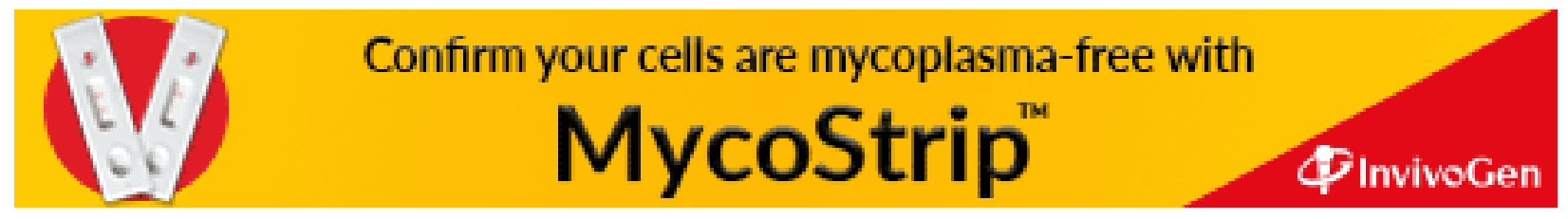

